\title{
A Novel Parameter-Adaptive VMD Method Based on Grey Wolf Optimization with Minimum Average Mutual Information for Incipient Fault Detection
}

\author{
Wang $X u^{1}$ and Jinfei $\mathrm{Hu} \mathbb{B D}^{2}$ \\ ${ }^{1}$ School of Marine Engineering Equipments, Zhejiang Ocean University, Zhoushan, Zhejiang 316022, China \\ ${ }^{2}$ National Engineering Research Center for Marine Aquaculture, Institute of Innovation and Application, \\ Zhejiang Ocean University, Zhoushan, Zhejiang 316022, China
}

Correspondence should be addressed to Jinfei Hu; hujinfei@zjou.edu.cn

Received 4 December 2020; Revised 18 January 2021; Accepted 22 January 2021; Published 2 February 2021

Academic Editor: Jinde Zheng

Copyright (c) $2021 \mathrm{Wang} \mathrm{Xu}$ and Jinfei $\mathrm{Hu}$. This is an open access article distributed under the Creative Commons Attribution License, which permits unrestricted use, distribution, and reproduction in any medium, provided the original work is properly cited.

\begin{abstract}
Recently, variational mode decomposition (VMD) has attracted wide attention on mechanical vibration signal analysis. However, there are still some dilemmas in the application of $\mathrm{VMD}$, such as the determination of the number of mode decomposition $\mathrm{K}$ and quadratic penalty term $\alpha$. In order to acquire appropriate parameters of VMD, an improved parameter-adaptive VMD method based on grey wolf optimizer (GWO) is developed by taking the minimum average mutual information into consideration (GWOMI). Firstly, the parameters $(K, \alpha)$ are adaptively determined through GWOMI. Then, the vibration signal is decomposed by the developed method and effective modes are extracted according to the maximum kurtosis. Finally, the extracted modes are processed by Hilbert envelope analysis to acquire the incipient fault features. With the simulation and experimental analysis, it is clearly found that the developed method is effective and performs better than some existing ones.
\end{abstract}

\section{Introduction}

Rotating machinery has been extensively employed in the manufacturing, traffic and transportation, marine vessel, etc. Rolling bearings are one of the most crucial parts of them and their unexpected failures usually lead to great production loss and high repair cost. Therefore, it is of great significance for fault detection at their incipient faulty stage to ensure the reliable and safely running of the machinery [1-4]. If a local fault occurs in a rolling bearing, some transient pulses will appear in the vibration signal. These transient pulses analysis can provide a possible solution for fault detection and diagnosis. However, these pulse signals caused by the early fault are usually very difficult for detection. Meanwhile, they are nonstationary, nonlinear, and easily covered by strong background noise, which makes it almost impossible to extract the incipient fault features from the raw signals $[5,6]$.
To address these problems, many methods have been developed based on signal processing. Time-frequency analysis is one of the most widely used signal processing methods to extract the fault feature. Wavelet transform is a typical time-frequency analysis method, which can decompose vibration signal into some wavelets and provide some significant local information. However, it is a nonadaptive signal analysis method as wavelet basis functions are determined in advance [7-11]. An empirical mode decomposition (EMD) method was developed by Huang et al. [12]. EMD could decompose a signal into some modes and a residue. And EMD has strong adaptive decomposition ability to time-varying signals [7]. Lu et al. [13, 14] investigated a kind of method based on an improved genetic algorithm and EMD, which could extract the fault features. However, there are some weaknesses of the methods based on EMD, such as poor antinoise capability and mode aliasing [15]. To address these above problems, Zheng et al. 
proposed a modified EMD approach, which was employed to process the nonstationary signal [16]. Ensemble empirical mode decomposition (EEMD) method was developed by Wu et al. [17]. In the work [18], this method was successfully used for the fault diagnosis of bearing signals. However, it would introduce new noises and reduce the computational efficiency. Zheng et al. put forward a partly EEMD method to overcome these problems [19].

VMD is a novel signal analysis method developed by Dragomiretskiy [20]. This method can decompose signals into some intrinsic mode functions, which have limited bandwidths and different center frequencies. It can overcome the shortcoming of mode aliasing and has been widely used in the fault diagnosis of rotating machinery. However, the effect of VMD relies on the choice of its parameters $(K$, $\alpha$ ). In practical applications, it is difficult to determine these parameters, and problems overdecomposition or underdecomposition may happen when parameters are not selected correctly $[21,22]$. Shen et al. developed a modified VMD based on initial center frequency, which was successfully used in the fault diagnosis of rotating machinery [23]. Zhang et al. utilized the grasshopper optimization algorithm to improve the parameter adaptiveness of VMD and this method was used to extract fault features successfully [24]. Zhu et al. developed an adaptive VMD method, which decomposed a complex signal into some band-limited intrinsic mode functions [25]. Zhao et al. utilized a single-objective salp swarm algorithm to optimize VMD parameters, which could reduce mode aliasing and kept the fidelity of complex vibration signals [26]. Gu et al. reported an adaptive VMD based on GWO. In this method, the minimum average envelope entropy was used as the objective function [3]. However, signal decomposition, whose parameters are obtained by the minimum average envelope entropy, may result in the loss of some fault features.

Inspired by the above research results, an improved parametric-adaptive VMD based on grey wolf optimizer with mutual information algorithm (GWOMI-VMD) is developed in this work. The GWO algorithm has a great global searching ability with high convergence accuracy. However, it is noted that the optimization objective function has a great impact on the optimization results. And there are still some overdecomposition or underdecomposition problems in the VMD method when the most commonly used objective functions are applied in GWO, such as smoothness index, sparsity measurement, and correlation coefficient [27]. Mutual information (MI) is a valuable information measure in information theory, which is used to represent the mutual dependence between two variables. In this paper, minimum average mutual information (MAMI) is selected as the optimization objective function to reflect the decomposition effect of VMD. Then, the parameters are optimized by GWOMI and the signal is decomposed by our proposed method. Finally, effective modes are extracted according to the maximum kurtosis and envelope spectrum analysis is applied to the effective modes to acquire fault features.

The remainder of this paper is organized as follows: the main principles of VMD and GWO are briefly introduced in Section 2. In Section 3, an improved parametric-adaptive VMD method based on GWO with mutual information is given in detail. In Section 4 and Section 5, both simulation and experimental signals are utilized to demonstrate the validity of the GWOMI-VMD, and some comparisons between the proposed method and traditional VMD, and particle swarm optimization (PSO) optimized VMD and EMD methods are provided. In Section 6, some conclusions are drawn.

\section{The Principle of VMD and GWO}

2.1. Variational Mode Decomposition. VMD is a novel method of signal processing, which adaptively decomposes signals into $K$ modes and these modes have different center frequencies $\omega_{k}$. The crucial issue of the VMD algorithm is how to acquire the solution of the constrained variational problem, which is formulated in equation (1) as follows:

$$
\begin{aligned}
& \min _{\left\{u_{k}\right\},\left\{\omega_{k}\right\}}\left\{\sum_{k}\left\|\partial_{t}\left[\left(\delta(t)+\frac{j}{\pi t}\right) \cdot u_{k}(t)\right] e^{-j \omega_{k} t}\right\|_{2}^{2}\right\}, \\
& \text { subject to } \sum_{k} u_{k}=f,
\end{aligned}
$$

where $\left\{\mathbf{u}_{k}\right\}=\left\{u_{1}, u_{2}, \ldots, u_{k}\right\}$ and $\left\{\omega_{k}\right\}$ represent each mode component and center frequency, respectively. We introduce Lagrange multiplier $\lambda$ and balance parameter $\alpha$ to transform equation (1) into an unconstrained one, which is formulated in the following equation:

$$
L\left(\left\{u_{k}\right\},\left\{\omega_{k}\right\}, \lambda\right)=\alpha \sum_{k}\left\|\partial_{t}\left[\left(\delta(t)+\frac{j}{\pi t}\right) \cdot u_{k}(t)\right] e^{-j \omega_{k} t}\right\|_{2}^{2}+\left\|f(t)-\sum_{k} u_{k}(t)\right\|_{2}^{2}+\left\langle\lambda(t), f(t)-\sum_{k} u_{k}(t)\right\rangle
$$

The alternating direction method of multipliers is employed to acquire the saddle point of equation (2). As shown in equations (3) and (4), $\widehat{u}_{k}^{n+1}$ and $\omega_{k}^{n+1}$ are updated to obtain the optimal values: 


$$
\begin{gathered}
\widehat{u}_{k}^{n+1}(\omega) \longleftarrow \frac{\hat{f}(\omega)-\sum_{i \neq k} u_{i}(\omega)+(\widehat{\lambda}(\omega) / 2)}{1+2 \alpha\left(\omega-\omega_{k}\right)^{2}}, \\
\omega_{k}^{n+1} \longleftarrow \frac{\int_{0}^{\infty} \omega\left\|\widehat{u}_{k}^{n+1}(\omega)\right\|^{2} \mathrm{~d} \omega}{\int_{0}^{\infty}\left\|\widehat{u}_{k}^{n+1}\right\|^{2} \mathrm{~d} \omega},
\end{gathered}
$$

where $n$ is the number of iterations.

After each update, the modes and corresponding center frequencies are obtained. After that, the Lagrange multiplier is updated as shown in the following equation:

$$
\hat{\lambda}^{n+1}(\omega) \longleftarrow \widehat{\lambda}^{n}(\omega)+\tau\left(\hat{f}(\omega)-\sum_{k} \widehat{u}_{k}^{n+1}(\omega)\right) .
$$

When equation (6) is satisfied, the above iteration is terminated:

$$
\sum_{k} \frac{\left\|\widehat{u}_{k}^{n+1}-\widehat{u}_{k}^{n}\right\|_{2}^{2}}{\left\|\widehat{u}_{k}^{n}\right\|_{2}^{2}}<\varepsilon .
$$

We can see from equation (3) that the modes are recognized as some Wiener filters. In equation (4), $\omega_{k}$ is the gravity of the corresponding mode power spectrum. And, in equation (2), the quadratic penalty term $\alpha$ can suppress mode aliasing, which is one of the critical parameters in VMD.

2.2. Grey Wolf Optimizer. Compared with other bionic intelligent algorithms, the grey wolf optimization algorithm has faster convergence and better global search capability. First, a group of grey wolves is randomly generated and they are divided into four social hierarchies according to their objective function values from best to worst, namely, $\alpha, \beta, \delta$, and $\omega$. Next, the hunting (optimization) is guided by $\alpha$, under the cooperation with $\beta$ and $\delta$ by the way of circling the prey. Then, $\omega$ and other wolves move towards the prey, periodically updating their position, gradually reducing the distance between them and the prey, and finally successfully hunting [28]. The main procedure of the algorithm is given as follows.

The distances between the wolves and their prey during the hunting are formulated in equations (7) and (8), respectively:

$$
\begin{aligned}
\vec{D} & =\left|\vec{h} \cdot \vec{X}_{P}(t)-\vec{X}_{t}\right|, \\
\vec{X}_{p}(t+1) & =\vec{X}_{p}(t)-\vec{A} \cdot \vec{D},
\end{aligned}
$$

where $\vec{X}_{p}(t), \vec{X}(t)$, and $t$ stand for prey position, wolf position, and iteration number, respectively. The coefficients $\vec{A}$ and $\vec{h}$ are calculated as follows:

$$
\begin{aligned}
& \vec{A}=2 \vec{a} \cdot \vec{n}_{1}-\vec{a}, \\
& \vec{h}=2 \cdot \vec{n}_{2},
\end{aligned}
$$

where $\vec{a}$ decreases gradually from 2 to 0 and $\vec{n}_{1}$ and $\vec{n}_{2}$ are random vectors in $[0,1]$.

During the hunting, $\alpha, \beta$, and $\delta$ are obtained for the first three optimal solutions in the optimization space, and the positions of $\beta$ and $\delta$ are changed according to $\alpha$. The specific process is shown as follows:

$$
\begin{aligned}
\vec{D}_{F} & =\left|\vec{h}_{i} \cdot \vec{X}_{F}(t)-\vec{X}(t)\right|, \\
\vec{X}_{i} & =\vec{X}_{F}-\vec{A}_{i} \cdot \vec{D}_{F}, \\
\vec{X}_{P}(t+1) & =\frac{\vec{X}_{1}+\vec{X}_{2}+\vec{X}_{3}}{3},
\end{aligned}
$$

where $F=\alpha, \beta, \delta$ and $i=1,2,3$.

\section{The Proposed Method}

3.1. Minimum Average Mutual Information. Mutual information (MI) is a nonparametric and nonlinear metric in information theory and can be used to reflect the mutual dependence between two variables. The expression is as follows:

$$
M I(a, b)=\sum_{a \in a} \sum_{b \in b} f(a, b) \lg \frac{f(a, b)}{f(a) f(b)},
$$

where $f(a, b)$ represents the joint probability density function of $a$ and $b$ and $f(a)$ and $f(b)$ are edge probability density functions of $a$ and $b$, respectively. If there is no overlapping information between $a$ and $b$, in other words, $a$ and $b$ are independent variables, the MI value between them is 0 . On the contrary, if there is more overlapping information between $a$ and $b$, the MI value is closer to 1 [29].

In this paper, the minimum average mutual information (MAMI) is selected as the optimization objective function and its expression is given by

$$
\text { objective }_{\mathrm{GWO}}=\operatorname{Minimum} \frac{\sum_{j=2}^{K} \mathbf{V}_{\mathrm{MI}(u(j-1), u(j))}^{\mathbf{u}_{\mathbf{k}}}}{K-1},
$$

where $K$ is the number of mode decomposition and $\sum_{j=2}^{K} \mathbf{V}_{M I(u(j-1), u(j))}^{\mathbf{u}_{\mathbf{k}}}$ represents the sum of MI between each neighboring mode. The smaller the MI is, the better the two modes are decomposed. Similarly, the smaller the average MI is, the better the overall effect of VMD will be. When $\left(\left(\sum_{j=2}^{K} \mathbf{V}_{\mathrm{MI}(u(j-1), u(j))}^{\mathbf{u}_{\mathbf{k}}}\right) /(K-1)\right)$ is minimized, the signal can be properly decomposed into a series of modes by VMD. At this moment, the corresponding parameters $(K, \alpha)$ are optimal.

3.2. Mode Extraction Based on Maximum Kurtosis. After the signal is processed by the GWOMI-VMD method, the effective mode is extracted according to the maximum kurtosis. Kurtosis describes the peak value of waveform and can reflect the numerical statistics of vibration signal distribution features [30]. The expression is as follows: 


$$
K=\frac{\sum_{i=1}^{n}\left(x_{i}-\mu\right)^{4}}{n \sigma^{4}}
$$

where $\mu$ is the mean of signal $\left[x_{1}, x_{2}, \ldots, x_{n}\right]$ and $\sigma$ and $n$ represent the standard deviation of the signal and the length of the signal, respectively.

As we all know, the more the impact components in the signal, the greater the corresponding kurtosis. Therefore, the effective mode with the maximum kurtosis is selected for the fault feature extraction.

3.3. GWOMI-VMD. In this paper, we develop an incipient fault characteristics extraction method from the rolling bearing vibration signal, named GWOMI-VMD. The steps of GWOMI-VMD are described briefly as follows:

(1) Input the vibration signal, set the parameter range of VMD parameters $K$ and $\alpha$, and initialize the parameters of GWO.

(2) Decompose the signal by VMD and calculate the average mutual information of the decomposed modes.

(3) Update the objective function value and record the position of the wolves.

(4) Judge whether the number of iterations exceeds the maximum number of iterations. If so, the iteration will be terminated. If not, go back to step 2 to continue the iteration.

(5) Obtain the minimum value of the optimization objective function and save the optimal position $(K$, $\alpha)$.

(6) The signal is decomposed by VMD with the optimized $(K, \alpha)$, and the effective mode is extracted based on the maximum kurtosis.

(7) The envelope spectrum of the extracted mode is calculated and the fault characteristics are correspondingly obtained.

The flowchart of the developed method is illustrated in Figure 1.

\section{Simulation Analysis}

In order to demonstrate the performance of the developed method, a simulation signal $y$ is configured as shown in the following equation:

$$
\left\{\begin{array}{l}
y_{1}(t)=\cos \left(2 * p i * f_{1} * t\right) \\
y_{2}(t)=\frac{1}{4}\left(\cos \left(2 * p i * f_{2} * t\right)\right) \\
y_{3}(t)=\frac{1}{16}\left(\cos \left(2 * p i * f_{3} * t\right)\right) \\
y=y_{1}+y_{2}+y_{3}+\text { noise }
\end{array}\right.
$$

where $f_{1}=2 \mathrm{~Hz}, f_{2}=24 \mathrm{~Hz}$, and $f_{3}=288 \mathrm{~Hz}$, the noise signal is random numbers distributed in $[-2,2]$, and the sampling frequency is $1000 \mathrm{~Hz}$. Figure 2 shows the composite signal $y$ in the time domain and its frequency spectrum. Figure 3 shows each component of the composite signal $y$. We can clearly see the frequency components $f_{1}$ and $f_{2}$ from the frequency spectrum, but the frequency component $f_{3}$ has been completely submerged by the random noises in Figure 2.

The proposed method is performed to search the optimal parameters of VMD. In the optimization process, the optimization objective function adopts the minimum average mutual information (MAMI). And the convergence diagram of the optimization objective function is illustrated in Figure 4. As seen from Figure 4, the objective function value is 0.017 when the iteration time reaches 14 , and, at this moment, the curve of the objective function converges. Besides, the corresponding parameters of VMD are optimized when the iteration time reaches 14 . Then, the optimized parameters $(9,4721)$ are substituted into VMD to decompose the composite signal $y$, and Figure 5 shows the resulting modes.

From Figure 5, we can see that three frequency components $f_{1}, f_{2}$, and $f_{3}$ are extracted, respectively, which are consistent with the frequencies of the simulation signal $y$. It is worth mentioning that there is no serious aliasing between these modes.

In addition, the simulation signal is processed by the traditional VMD with parameters $(3,2000)$ as shown in Figure 6 . We can clearly see that both frequency components $f_{1}$ and $f_{2}$ are extracted in the first mode. However, the frequency component $f_{3}$ cannot be extracted from these modes. Lastly, the particle swarm optimization (PSO) is utilized to improve the traditional VMD and optimal parameters $(10,4883)$ are obtained correspondingly. Then, the composite signal $y$ is decomposed and the decomposition results are illustrated in Figure 7. From Figure 7, we can see that frequency components $f_{1}$ and $f_{2}$ are also extracted, but frequency component $f_{3}$ cannot be successfully extracted.

Through the above comparison analysis, we can find that the three frequency components $f_{1}, f_{2}$, and $f_{3}$ can be extracted accurately through the proposed method. However, the traditional VMD and PSO-VMD could not accurately extract the frequency component $f_{3}$. So, it is obvious that our proposed method GWOMI-VMD has a better performance than the traditional VMD and PSOVMD.

\section{Experiment Analysis}

The validity and superiority of the GWOMI-VMD in weak fault diagnosis of rolling bearing are demonstrated through analyzing the experimental signal. The experimental data comes from the Case Western Reserve University Bearing Data Center [31]. Figure 8 shows the basic layout of the bearing test table.

As seen from Figure 8, the test table consists of the following parts: a dynamometer, an electric motor, and a torque transducer/encoder. The tested bearing supports the motor spindle shaft, and the tested bearing has single point faults. The vibration signal is collected through an 


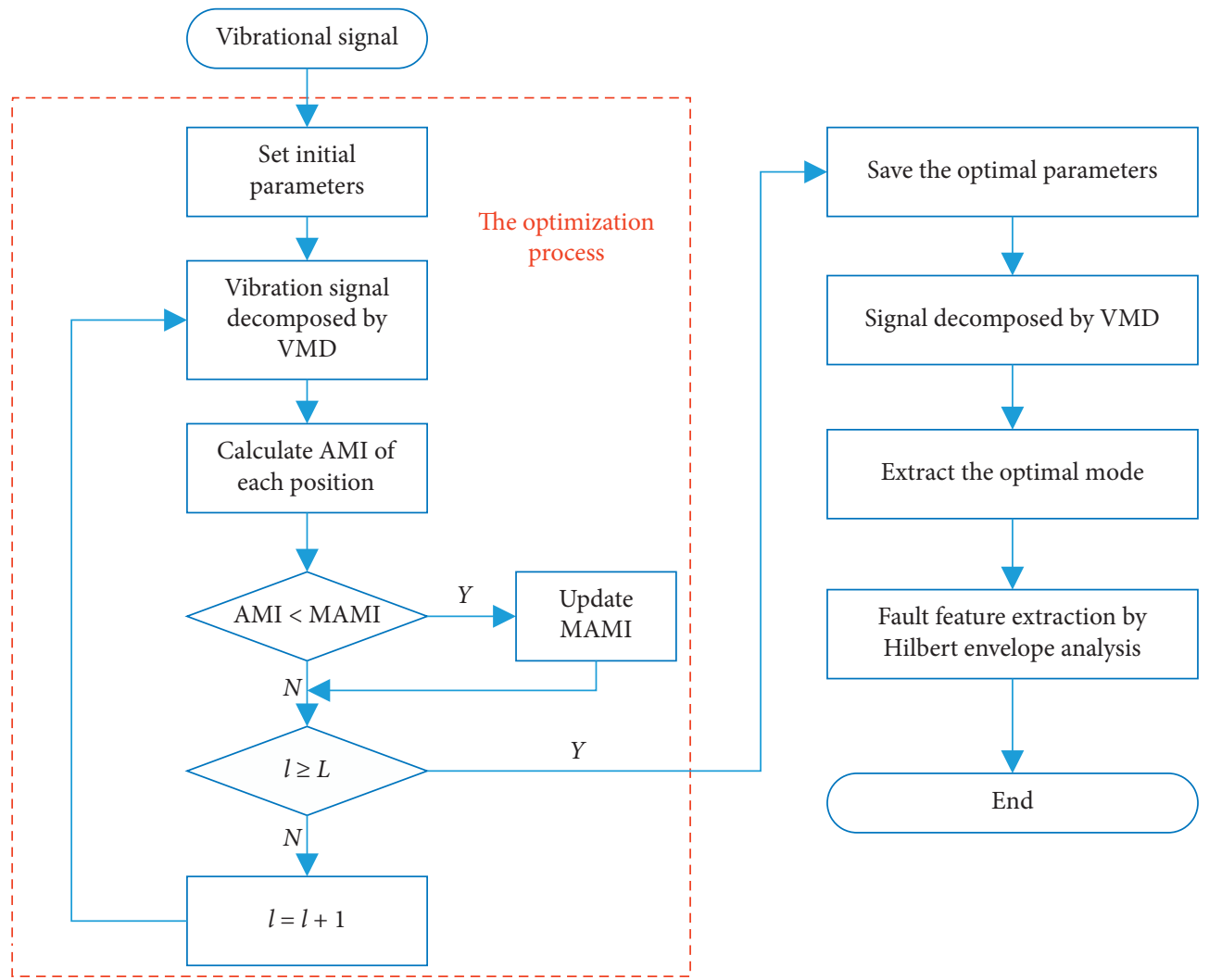

Figure 1: Procedure of the GWOMI-VMD.

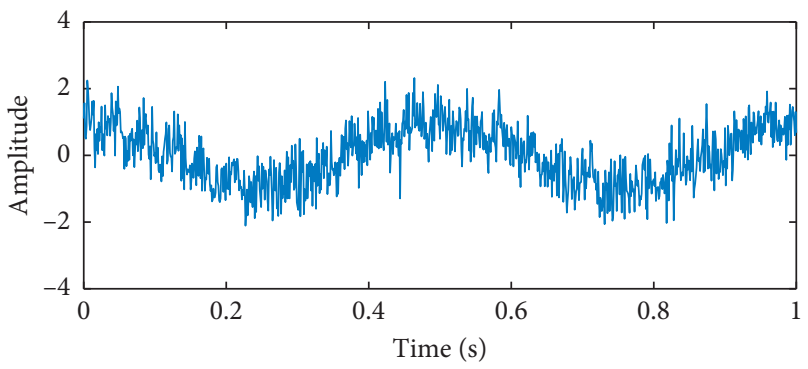

(a)

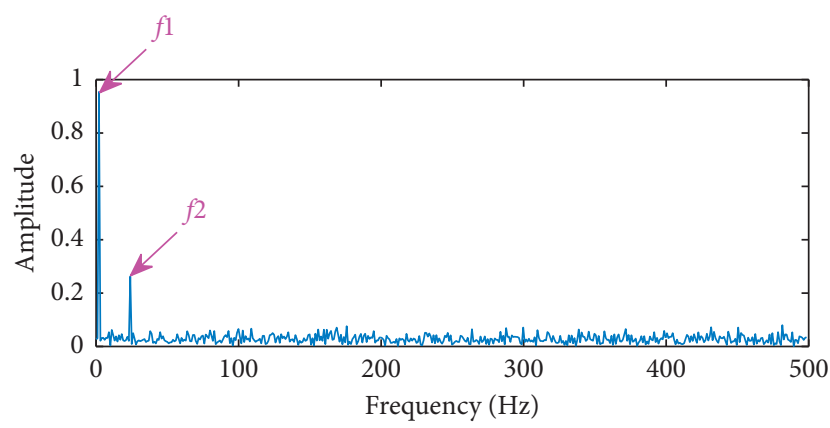

(b)

Figure 2: Simulation signal (y): (a) time-domain waveform and (b) frequency spectrum.

accelerometer. The specific technical parameters are listed in Table 1 and 6000 data points are sampled for analysis.

5.1. Bearing Inner Race Fault Feature Extraction. Figure 9 shows the experimental signal and it is processed based on the GWOMI-VMD method. Firstly, the optimal parameters $(K, \alpha)$ are determined according to the GWOMI algorithm. Figure 10 shows the GWOMI and PSO convergence curves for VMD parameter optimization. When the number of iterations is 13 , PSO starts to converge at the objective function value of 0.032896 . When the number of GWOMI iterations is 7 , the objective function value is 0.032876 , and the corresponding parameters of VMD are optimal with a value of $(10,5612)$. We can find that the convergence accuracy and speed of GWOMI are obviously better than those of PSO.

Then, the optimized parameters are substituted into VMD, and the signal is decomposed. Figure 11 shows the modes acquired by decomposition using GWOMI-VMD. The mode, which contains the most useful fault information, cannot be judged intuitively from these modes. Therefore, an index needs to be determined to help extract the effective mode.

As described above, the maximum kurtosis is used as an indicator for mode extraction in this work. The kurtosis diagram of the ten modes is shown in Figure 12. We can see that the kurtosis of the $10^{\text {th }}$ mode is the highest out of them, with a value of 3.113 . Therefore, we choose the $10^{\text {th }}$ mode for the following analysis. 


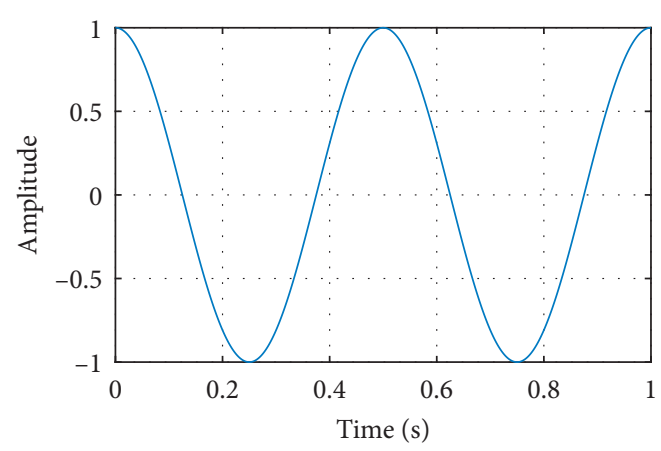

(a)

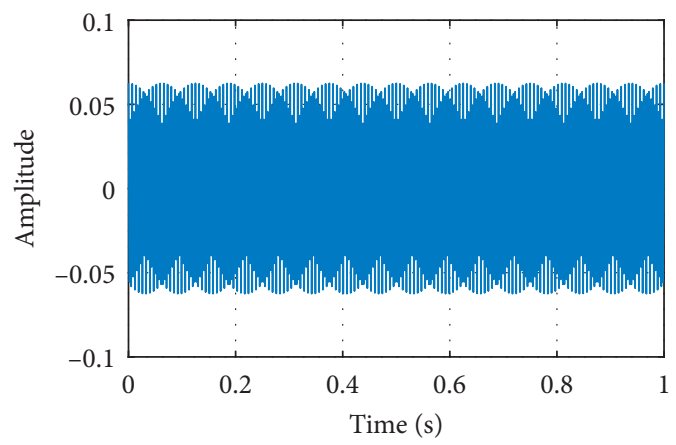

(c)

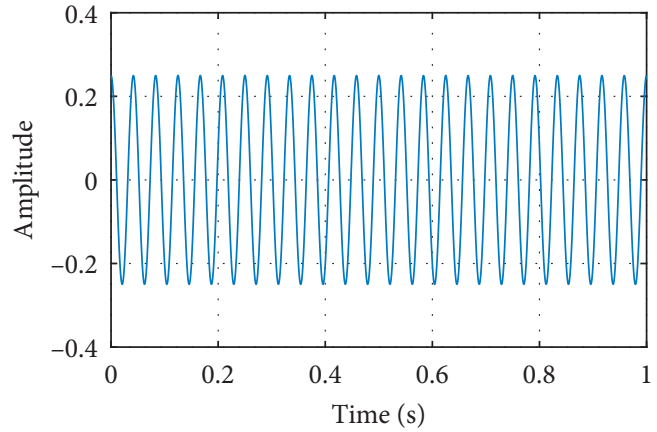

(b)

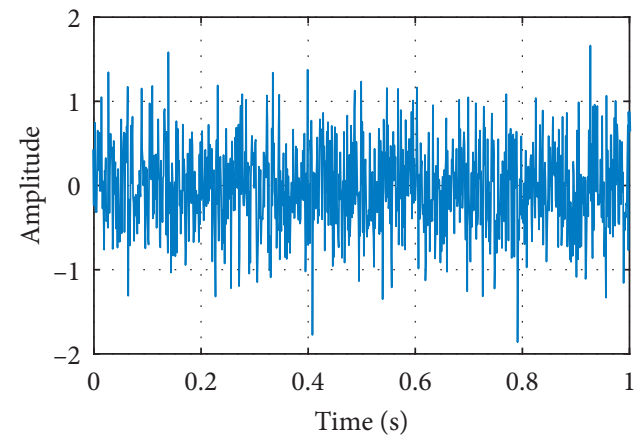

(d)

FiguRE 3: Each part of the simulation signal: (a) harmonic signal $y_{1}$, (b) harmonic signal $y_{2}$, (c) harmonic signal $y_{3}$, and (d) noise signal.

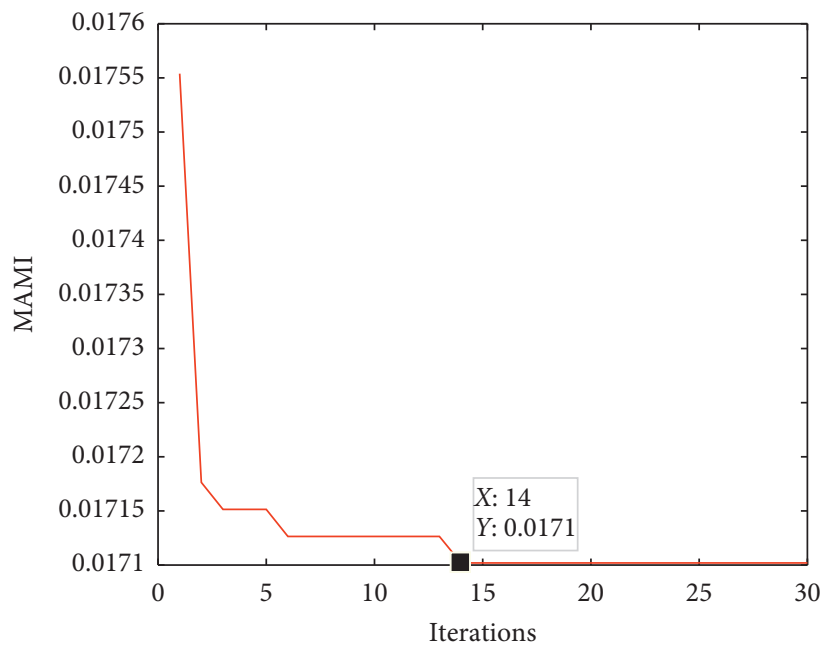

FIGURE 4: Convergence curve of GWOMI.

Envelope spectrum analysis is performed on the $10^{\text {th }}$ mode and Figure 13 shows the results of the analysis. From Figure 13, the fault frequency $f$, rotation frequency fr, and its second harmonic frequency $2 f r$ have emerged obviously. What is more, compared with the frequency spectrum in Figure 9, we can find that the background noise is basically eliminated in Figure 13.

Comparison analysis: a performance comparison between maximum kurtosis and maximum correlation coefficient as the mode index is conducted. Figure 14 shows the envelope spectrum of the effective mode, which was extracted based on the maximum correlation coefficient. We find that although fault frequency $f$ is extracted, the rotational speed frequency fr is not extracted as shown in Figure 13. In addition, there is a newly generated interference frequency, whose amplitude in the spectrum exceeds the fault frequency $f$.

In addition, the GWOMI-VMD is further compared with traditional VMD. In this paper, the parameters of traditional VMD are set as $(5,2500)$ based on experience. The 

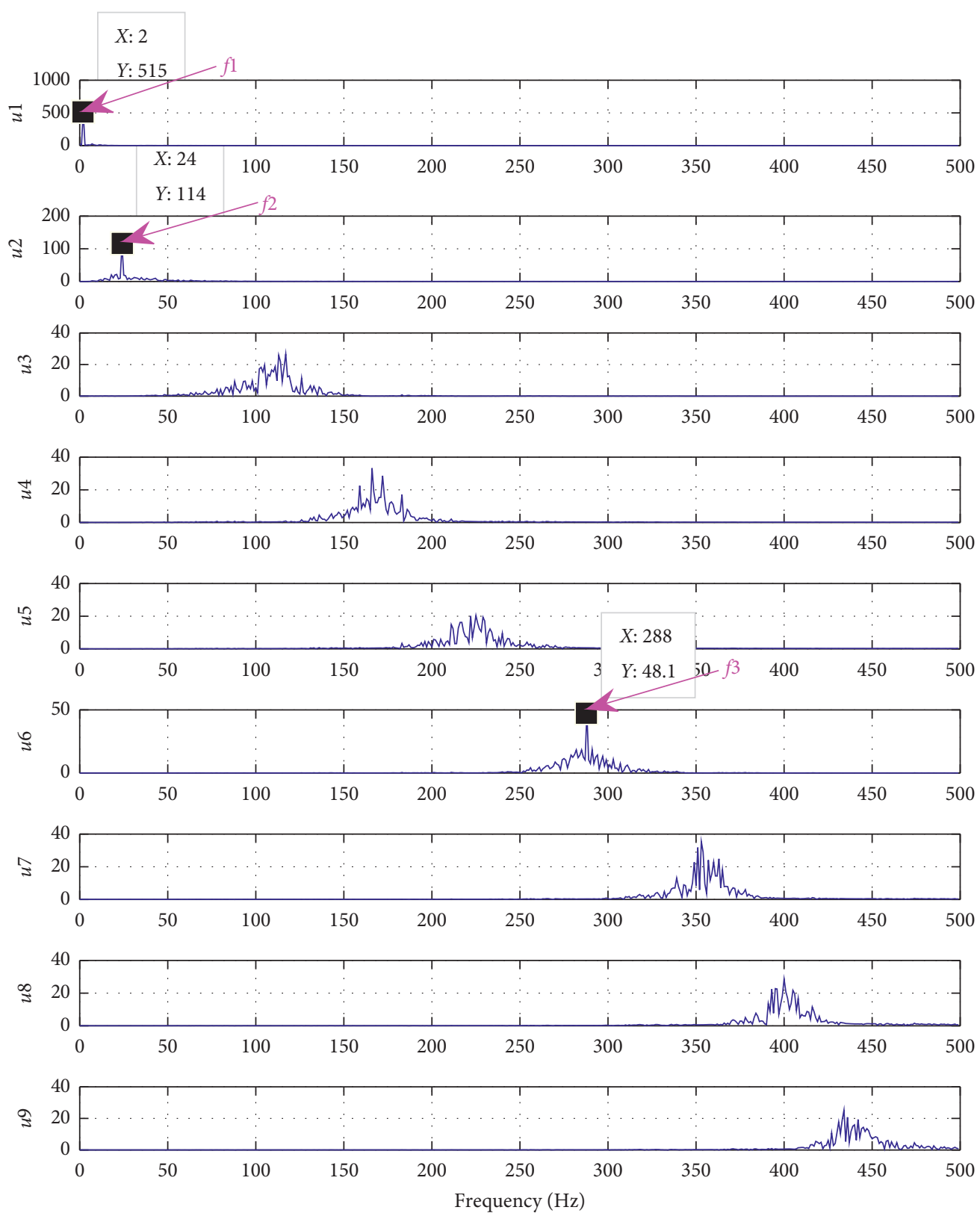

FIgURE 5: The spectrum of each mode decomposed by the proposed method.

decomposition results of traditional VMD are shown in Figure 15. Then, GWOMI-VMD method is compared with the EMD method and Figure 16(a) shows the first five modes obtained by using the GWOMI-VMD method. Figure 16(b) shows the first five modes obtained by using the EMD method. As seen from Figures 15 and 16(b), there is a certain degree of aliasing between these modes in the case of traditional VMD and EMD.

5.2. Bearing Outer Race Fault Feature Extraction. As demonstrated in the inner race, the optimal parameter of VMD is first obtained with the value of $(9,4340)$, and then the effective mode is extracted according to the maximum kurtosis. The kurtosis of the $9^{\text {th }}$ mode is the highest, with a value of 6.1024 . Therefore, the $9^{\text {th }}$ mode is extracted out and Figure 17(a) shows its envelope spectrum. The frequency components in Figure 17(a) are basically consistent with the calculated fault frequencies listed in Table 1.

A performance comparison between maximum kurtosis and maximum correlation coefficient both as the mode index is conducted. Figure 17(b) gives the spectrum of the effective mode extracted by the maximum correlation coefficient. From Figure 17(b), we can find that the second rotation harmonic frequency $2 \mathrm{fr}$ is extracted. However, it is obvious that the fault frequency $f$ is not extracted successfully, compared with Figure 17(a). Therefore, the effective mode extracted by the maximum kurtosis utilized in this paper has certain advantages over the correlation coefficient. 

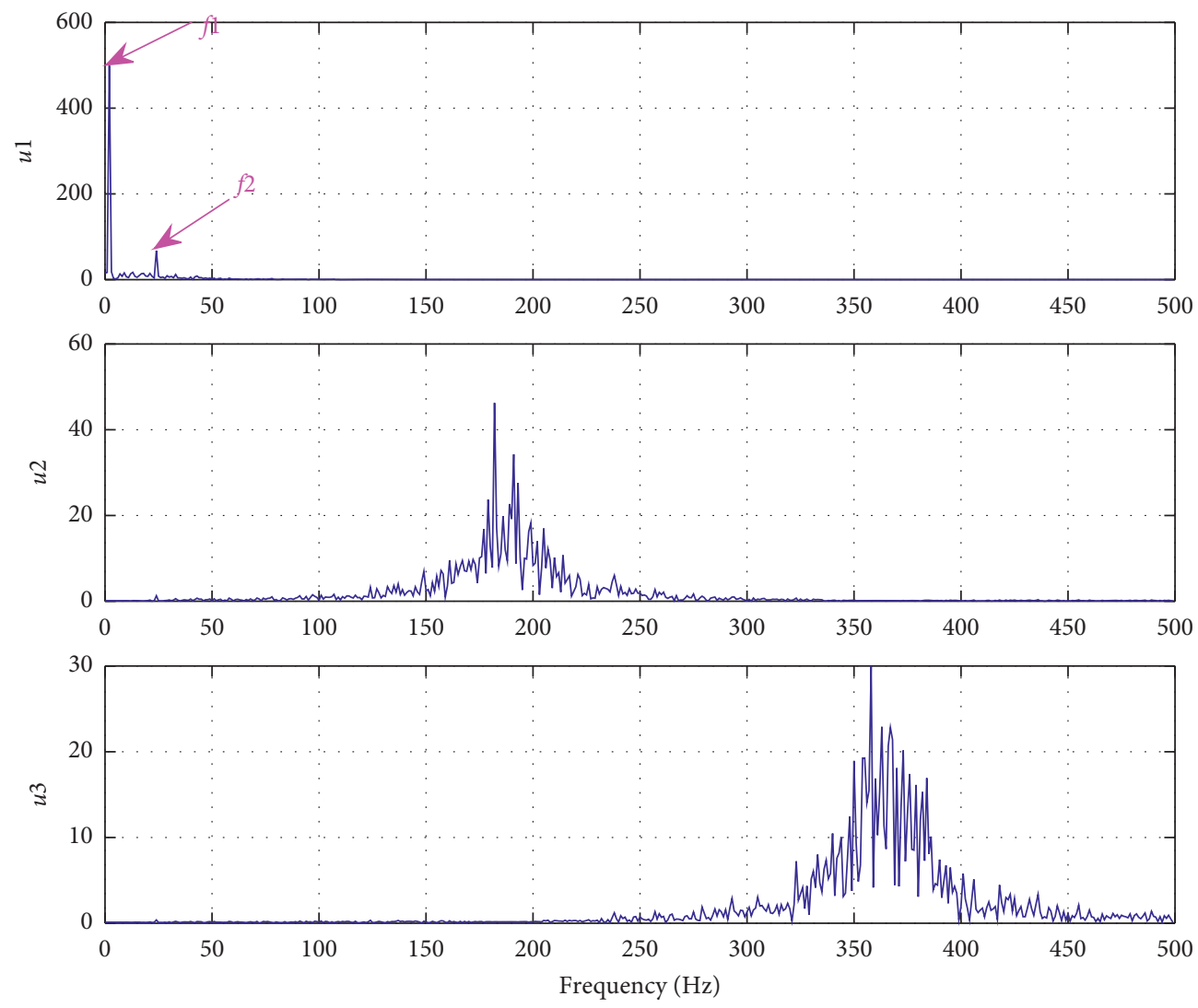

FIGURE 6: The spectrum of each mode decomposed by traditional VMD.
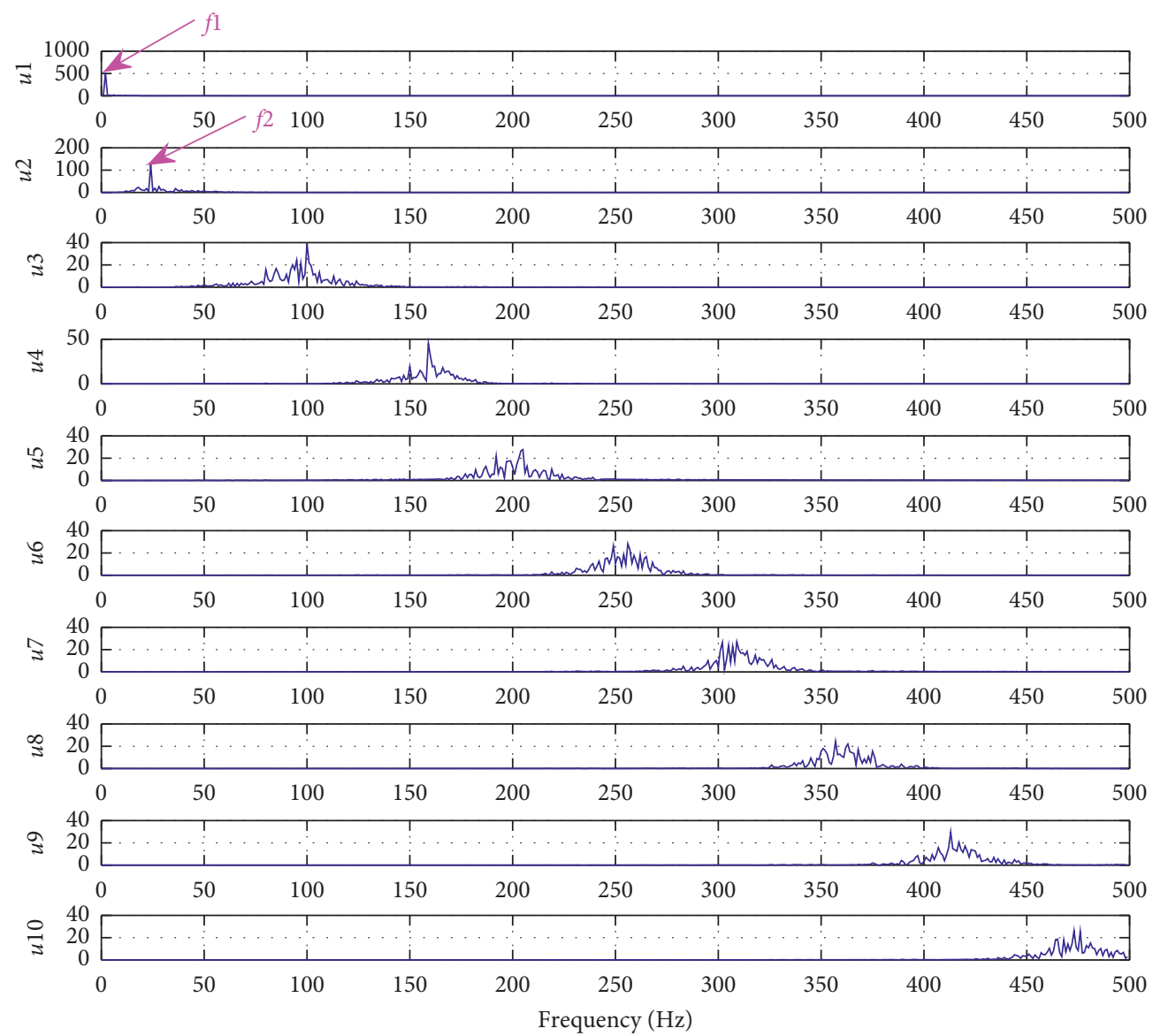

FIgURe 7: The spectrum of each mode decomposed by PSO-VMD. 


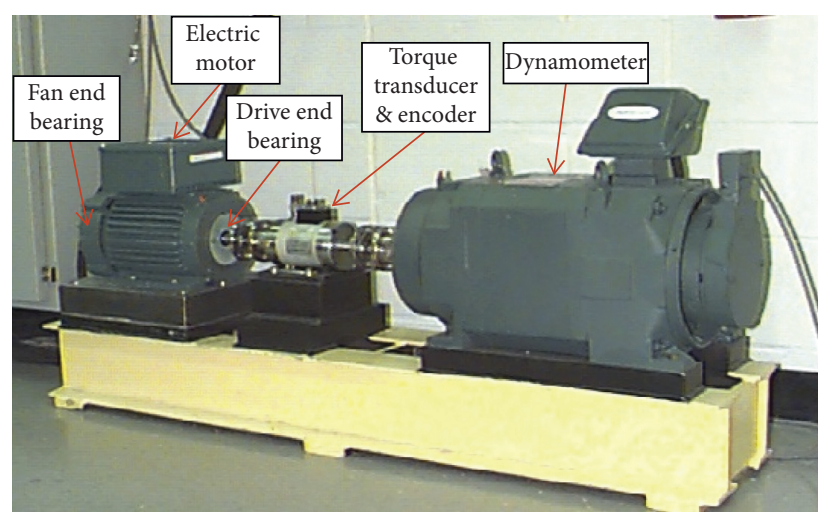

Figure 8: CWRU bearing test stand.

TABle 1: Technical parameters.

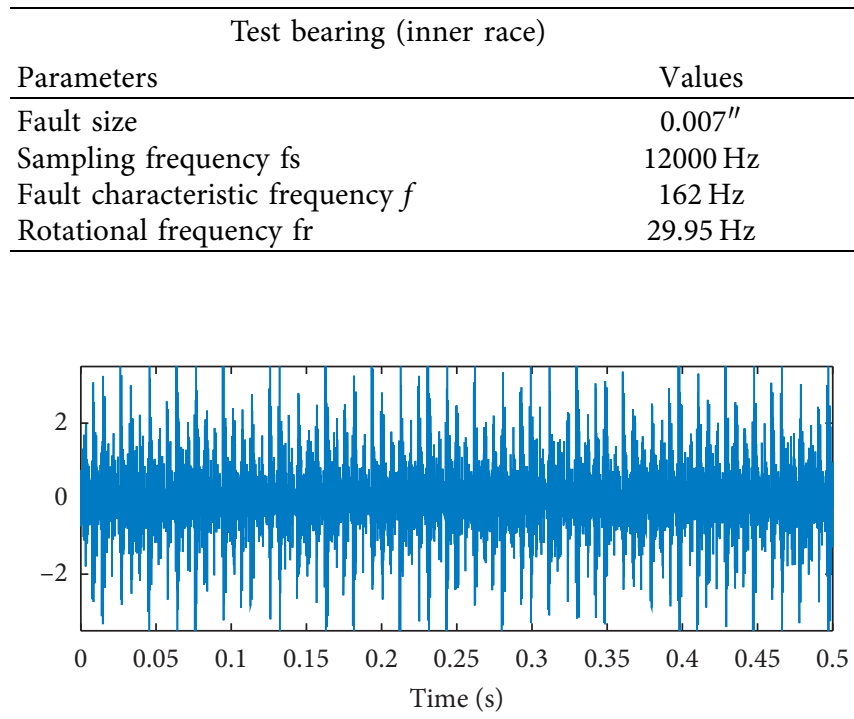

(a)

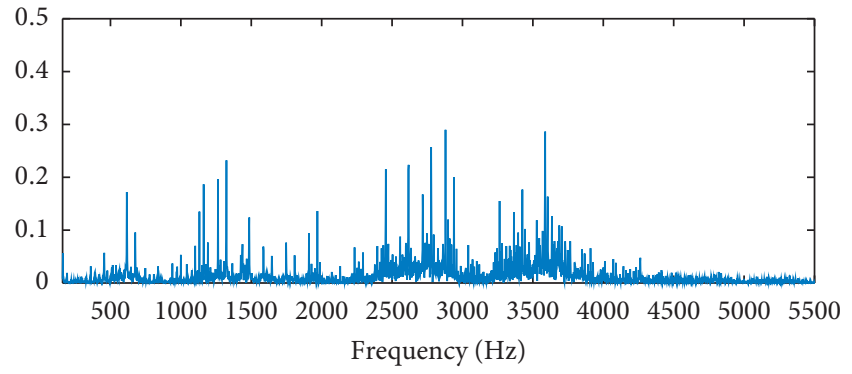

(b)

FIgURE 9: The original signal (a) time domain and (b) frequency domain.

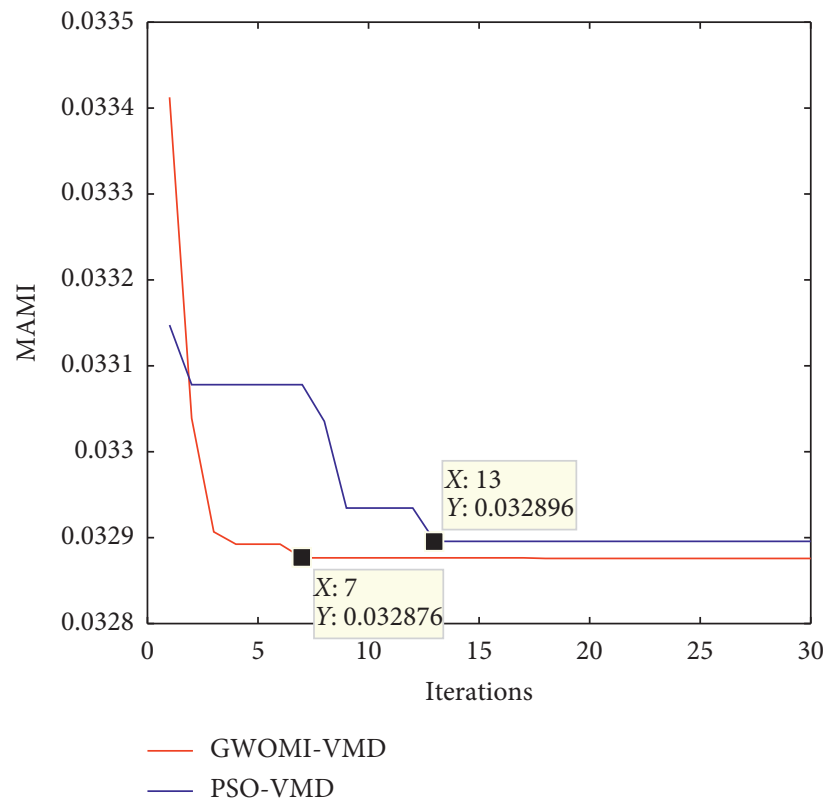

Figure 10: Convergence curves of GWOMI and PSO. 


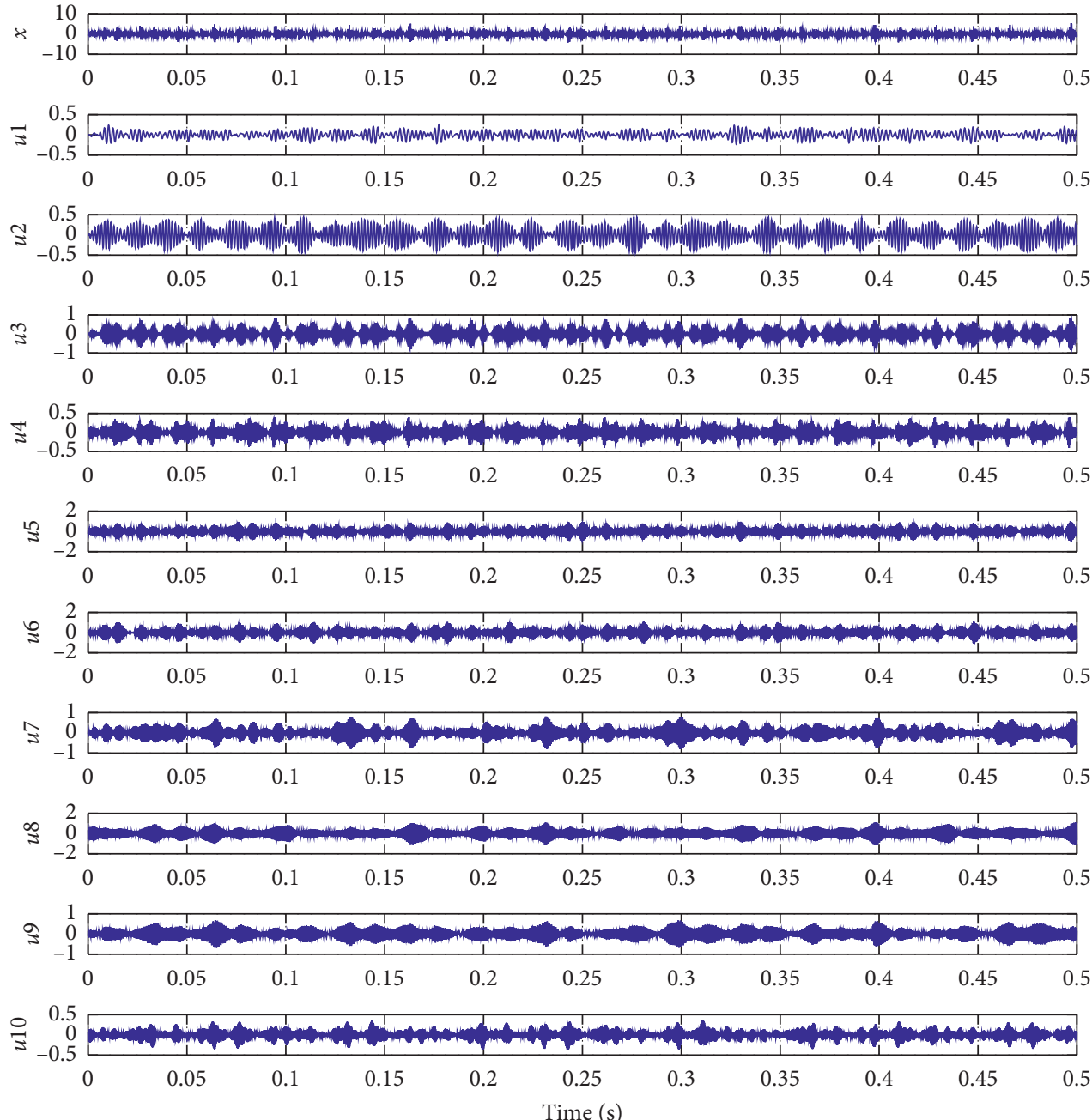

FIGURE 11: Results of the proposed method.

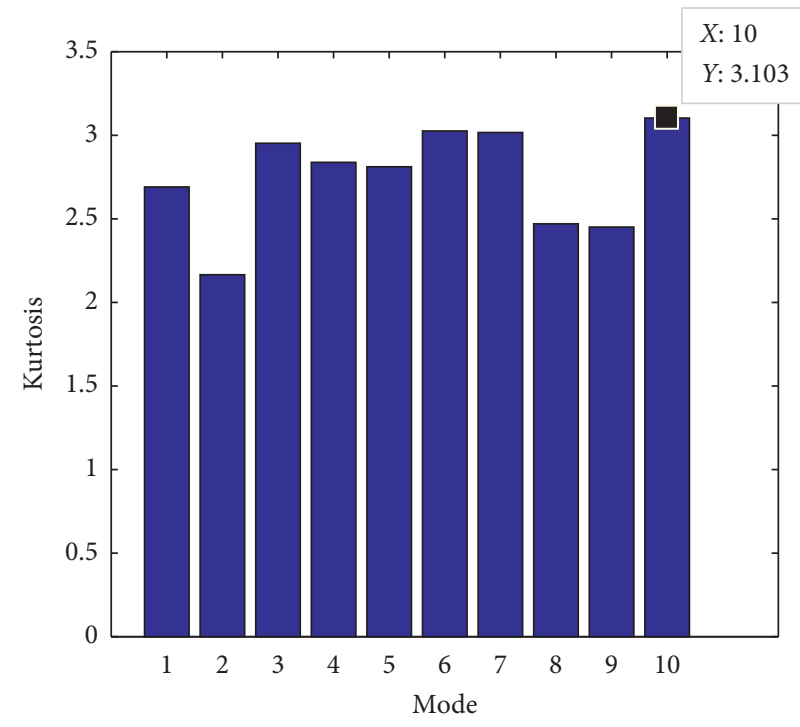

FIGURE 12: Kurtosis values of each mode. 


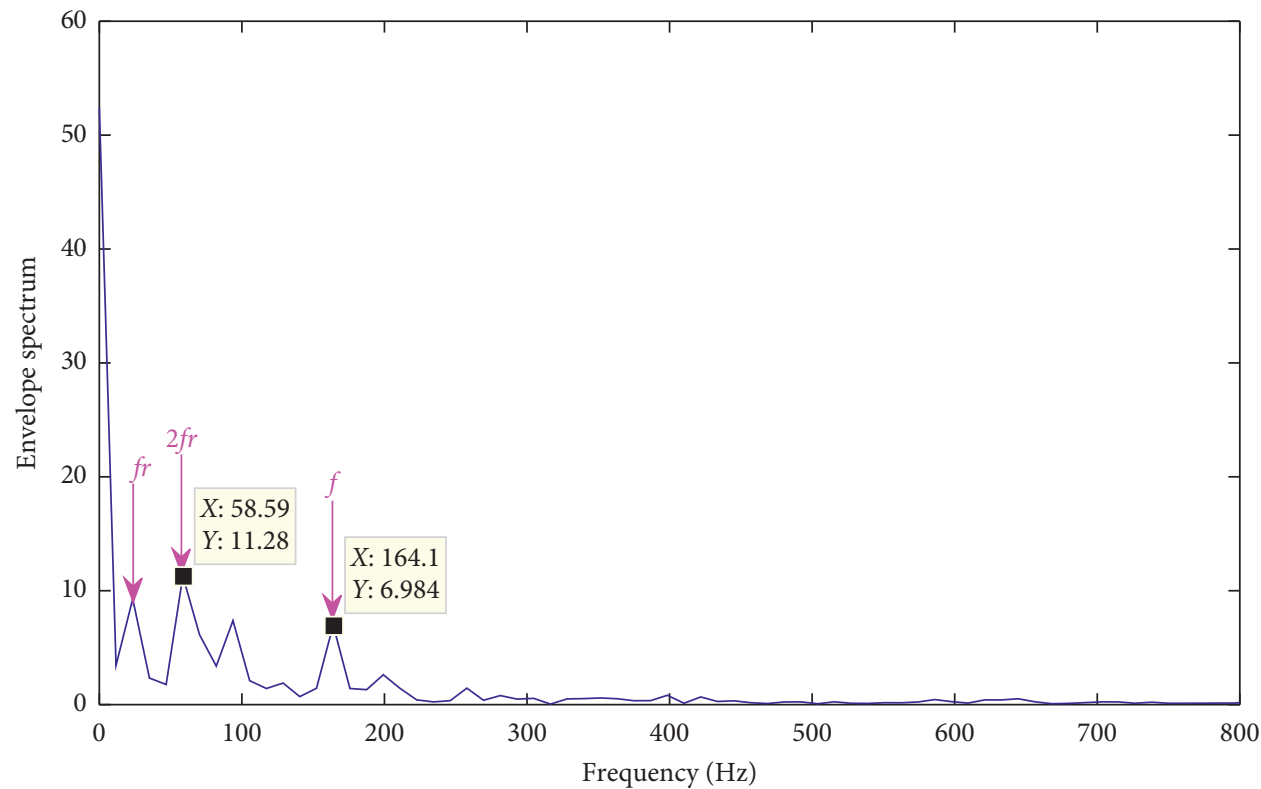

FIGURE 13: Envelope spectrum of effective mode extracted based on the maximum kurtosis.

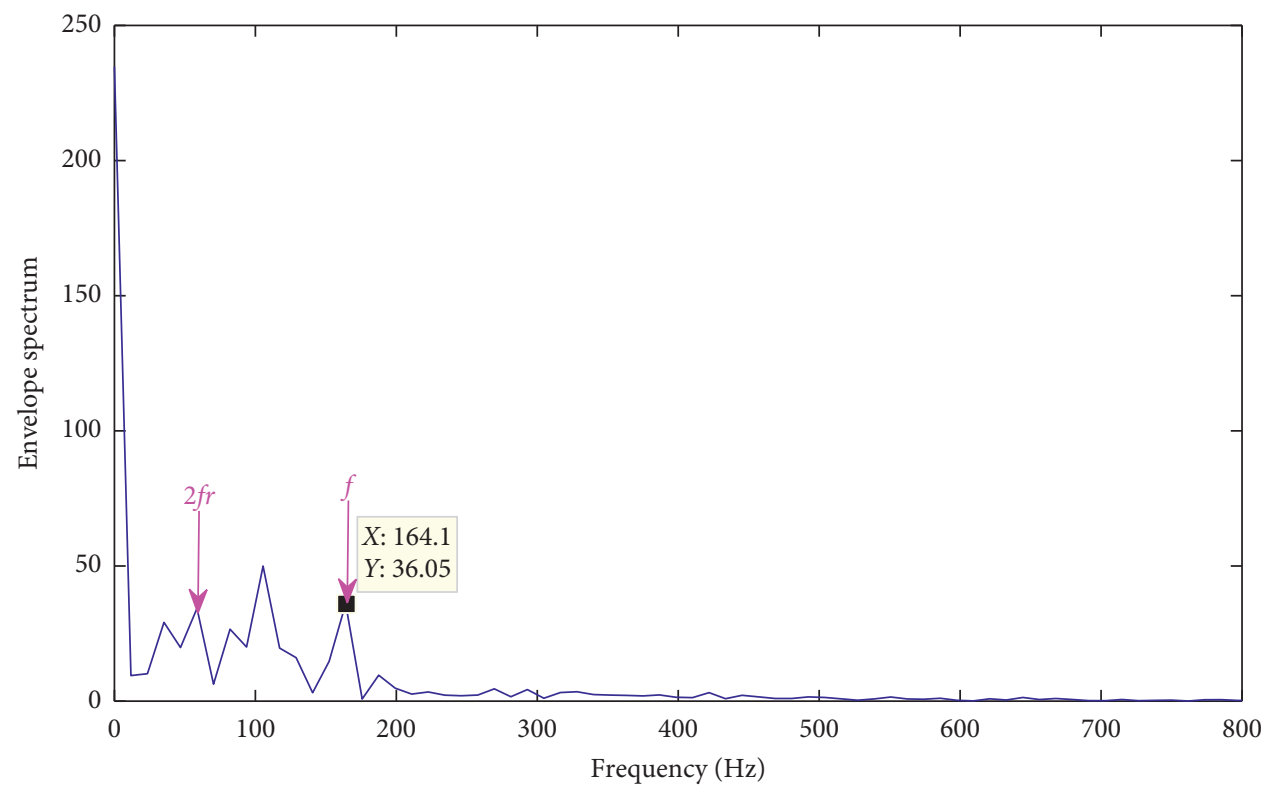

FIgURE 14: Envelope spectrum of effective mode extracted according to the maximum correlation coefficient. 

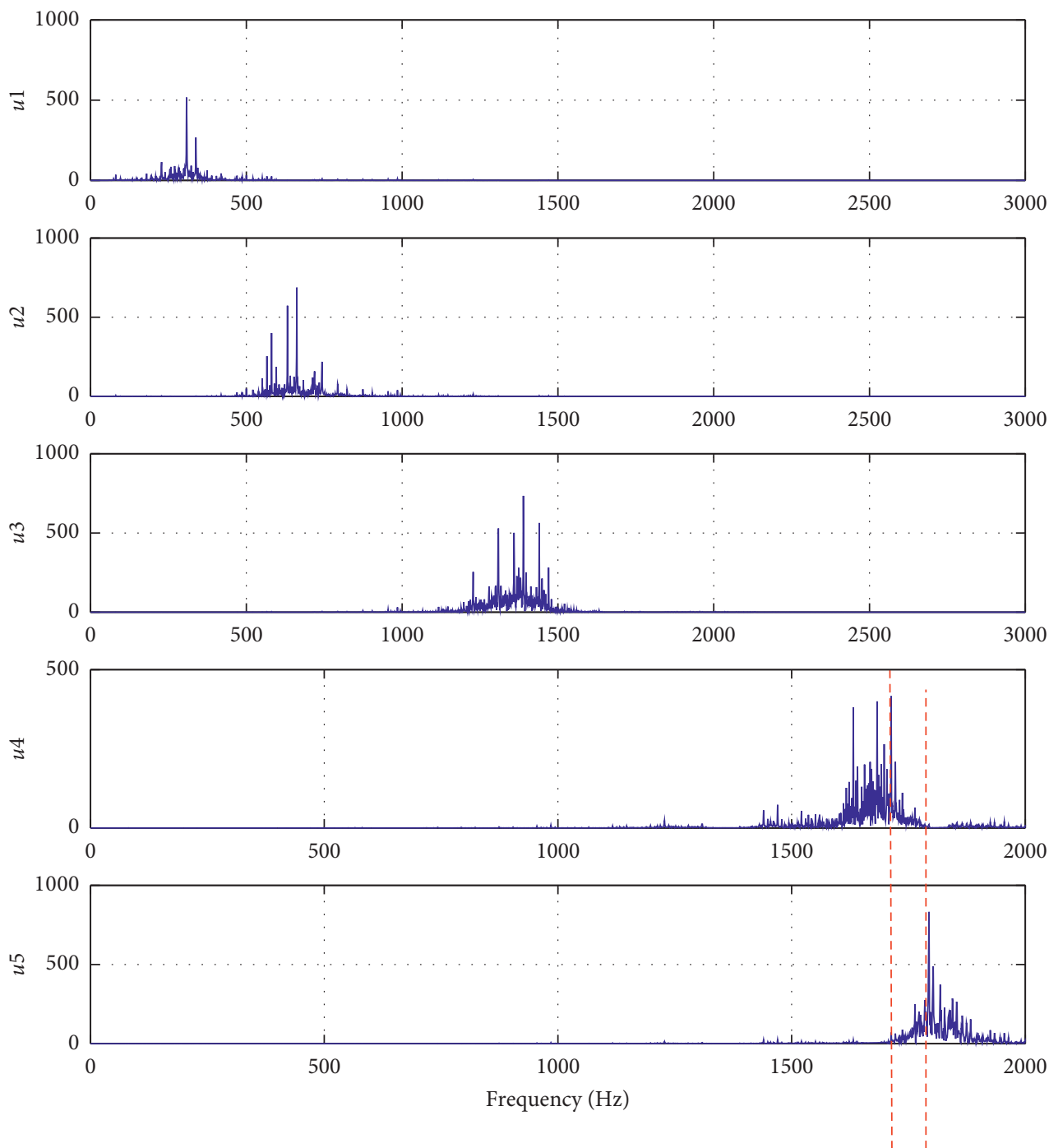

FIGURE 15: Spectrums of each mode decomposed by traditional VMD.
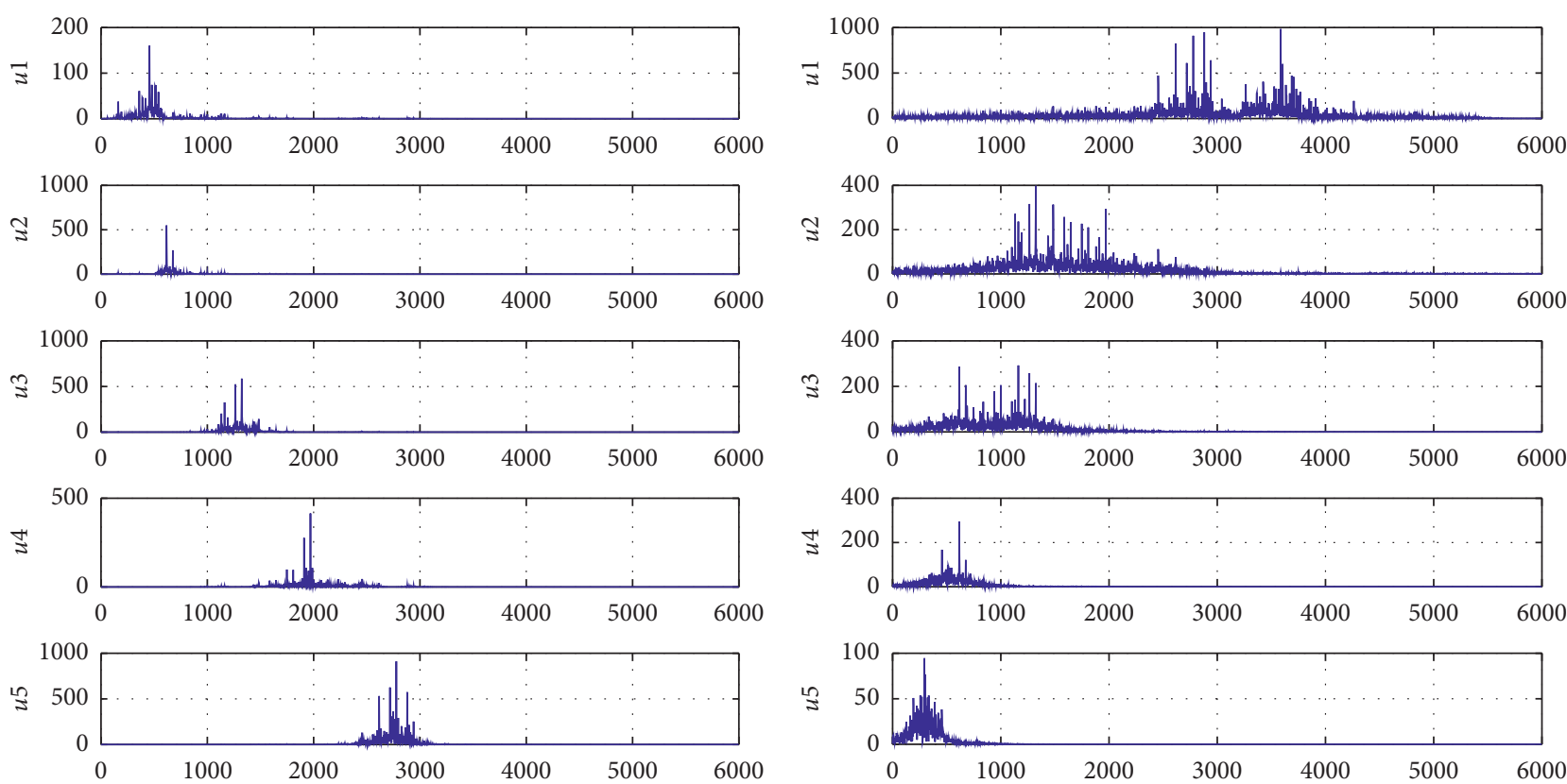

(a)

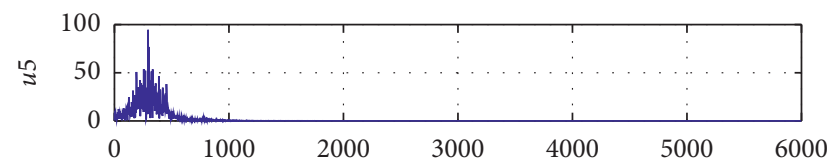

(b)

FIgURE 16: Frequency spectrums of $u 1, u 2, u 3, u 4$, and $u 5$ decomposed by (a) GWOMI-VMD and (b) EMD. 


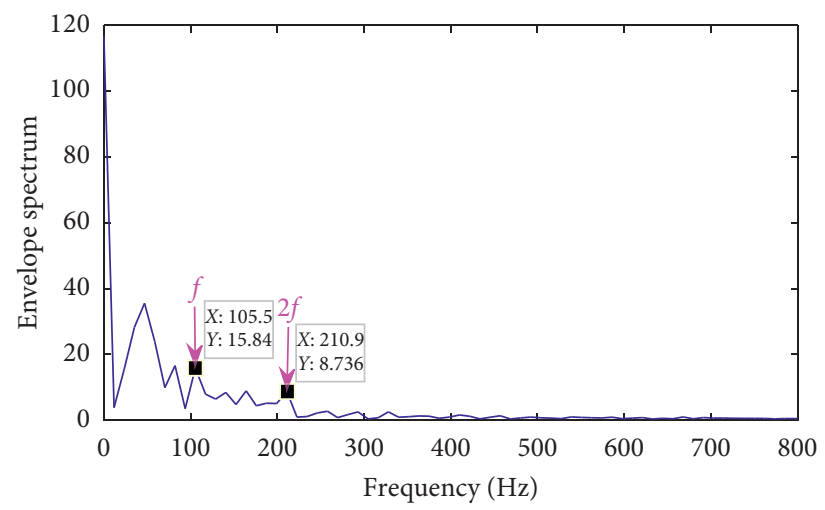

(a)

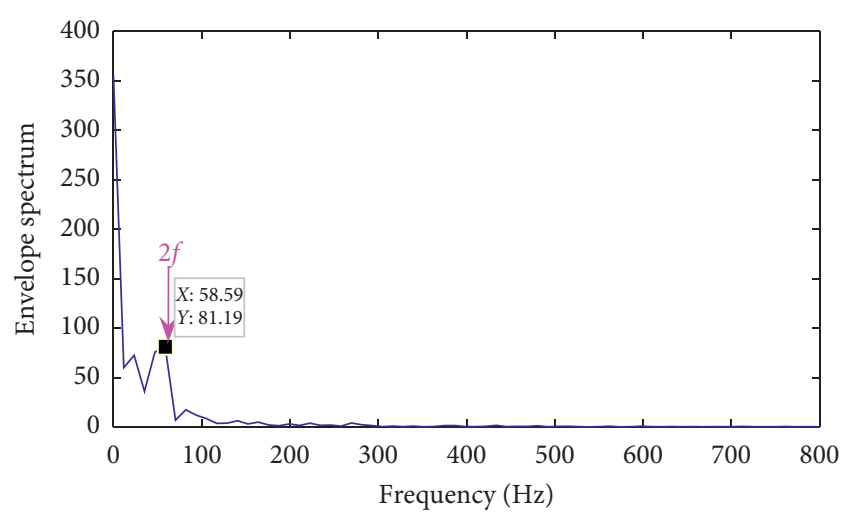

(b)

FIGURE 17: Envelope spectrum of effective mode extracted by (a) the maximum kurtosis and (b) the maximum correlation coefficient.

\section{Conclusions}

A parameter-adaptive VMD method based on GWOMI for fault diagnosis of rolling bearing is proposed in this work. It greatly enhances the parameter-adaptive ability of VMD. Firstly, the minimum mean mutual information is used as the optimization objective function of the GWOMI-VMD. After that, the maximum kurtosis is set as the effective mode index. Finally, envelope spectrum analysis is implemented on the effective mode to extract the fault feature. Through simulation and experimental analysis, the validity and feasibility of GWOMI-VMD are demonstrated. In addition, comparison results between GWOMI-VMD and the traditional VMD, PSO-VMD, and EMD are provided, from which the superiority of the GWOMI-VMD is further verified. Therefore, this paper has some certain values for incipient fault detection and diagnosis of rotating machinery.

\section{Data Availability}

The experimental data come from the Case Western Reserve University Bearing Data Center.

\section{Conflicts of Interest}

The authors declare that they have no known competing financial interests or personal relationships that could have appeared to influence the work reported in this paper.

\section{Acknowledgments}

This research was supported in part by the Natural Science Foundation of Zhejiang Province, China, under Grant LQ18F010009. And this research was also supported by the Fundamental Research Funds for Zhejiang Provincial Universities and Research Institutes (no. 2019J00046).

\section{References}

[1] J. Jiao, J. H. Yue, D. Pei, and Z. Q. Hu, “Application of feature fusion using coaxial vibration signal for diagnosis of rolling element bearings," Shock and Vibration, vol. 2020, Article ID 8831723, 14 pages, 2020.
[2] C. Q. Shen, Y. M. Qi, J. Wang et al., "An automatic and robust features learning method for rotating machinery fault diagnosis based on contractive autoencoder," Engineering Applications of Artificial Intelligence, vol. 76, pp. 170-184, 2018.

[3] R. Gu, J. Chen, R. J. Hong et al., "Incipient fault diagnosis of rolling bearings based on adaptive variational mode decomposition and Teager energy operator," Measurement, vol. 149, Article ID 106941, 13 pages, 2020.

[4] X. Yan and M. Jia, "Application of CSA-VMD and optimal scale morphological slice bispectrum in enhancing outer race fault detection of rolling element bearings," Mechanical Systems and Signal Processing, vol. 122, pp. 56-86, 2019.

[5] Y. Pan, R. Hong, J. Chen, Z. Qin, and Y. Feng, "Incipient fault detection of wind turbine large-size slewing bearing based on circular domain,” Measurement, vol. 137, pp. 130-142, 2019.

[6] H. Cao, F. Fan, K. Zhou, and Z. He, "Wheel-bearing fault diagnosis of trains using empirical wavelet transform," Measurement, vol. 82, pp. 439-449, 2016.

[7] X. Zhang, Q. Miao, Z. Liu, and Z. He, "An adaptive stochastic resonance method based on grey wolf optimizer algorithm and its application to machinery fault diagnosis," ISA Transactions, vol. 71, no. 2, pp. 206-214, 2017.

[8] X. Chen, Y. Yang, Z. Cui, and J. Shen, "Vibration fault diagnosis of wind turbines based on variational mode decomposition and energy entropy," Energy, vol. 174, pp. 1100-1109, 2019.

[9] H. Yu, H. Li, and Y. Li, "Vibration signal fusion using improved empirical wavelet transform and variance contribution rate for weak fault detection of hydraulic pumps," ISA Transactions, vol. 107, pp. 385-401, 2020.

[10] A. Kurmar, Y. Q. Zhou, C. P. Gandhi et al., "Bearing defect size assessment using wavelet transform based Deep Convolutional Neural Network (DCNN)," Alexandria Engineering Journal, vol. 29, no. 2, pp. 999-1012, 2020.

[11] J. Chen, Z. Li, J. Pan et al., "Wavelet transform based on inner product in fault diagnosis of rotating machinery: a review," $\mathrm{Me}$ chanical Systems and Signal Processing, vol. 70-71, pp. 1-35, 2016.

[12] Z. Shen, Q. A. Zheng, M. L. Wu et al., "The empirical mode decomposition and the Hilbert spectrum for nonlinear and non-stationary time series analysis," Proceedings of the Royal Society A: Mathematical, Physical and Engineering Sciences, vol. 454, no. 1971, pp. 903-995, 1998.

[13] L. Lu, J. Yan, and C. W. De Silva, "Dominant feature selection for the fault diagnosis of rotary machines using modified 
genetic algorithm and empirical mode decomposition," Journal of Sound and Vibration, vol. 344, pp. 464-483, 2015.

[14] Y. Xue, D. Y. Dou, and J. G. Yang, "Multi-fault diagnosis of rotating machinery based on deep convolution neural network and support vector machine," Measurement, vol. 156, Article ID 107571, 7 pages, 2020.

[15] X. Yuan, Q. Tan, X. Lei, Y. Yuan, and X. Wu, "Wind power prediction using hybrid autoregressive fractionally integrated moving average and least square support vector machine," Energy, vol. 129, pp. 122-137, 2017.

[16] J. D. Zheng and H. Y. Pan, "Mean-optimized mode decomposition: an improved EMD approach for non-stationary signal processing," ISA Transactions, vol. 106, pp. 392-401, 2020.

[17] Z. Wu and N. E. Huang, "Ensemble empirical mode decomposition: a noise-assisted data analysis method," $A d$ vances in Adaptive Data Analysis, vol. 1, no. 1, pp. 1-41, 2009.

[18] M. E. Torres, M. A. Colominas, and G. Schlotthauer, "A complete ensemble empirical mode decomposition with adaptive noise," in Proceedings of the IEEE International Conference on Acoustics IEEE, Prague Congress Centre (PCC), pp. 4144-4147, Prague, Czech Republic, 2011.

[19] J. Zheng, J. Cheng, and Y. Yang, "Partly ensemble empirical mode decomposition: an improved noise-assisted method for eliminating mode mixing," Signal Processing, vol. 96, pp. 362-374, 2014.

[20] K. Dragomiretskiy and D. Zosso, "Variational mode decomposition," IEEE Transactions on Signal Processing, vol. 62, no. 3, pp. 531-544, 2014.

[21] M. Zhang, Z. Jiang, and K. Feng, "Research on variational mode decomposition in rolling bearings fault diagnosis of the multistage centrifugal pump," Mechanical Systems and Signal Processing, vol. 93, pp. 460-493, 2017.

[22] Z. Li, J. Chen, Y. Zi, and J. Pan, "Independence-oriented VMD to identify fault feature for wheel set bearing fault diagnosis of high speed locomotive," Mechanical Systems and Signal Processing, vol. 85, pp. 512-529, 2017.

[23] X. Jiang, C. Shen, J. Shi, and Z. Zhu, "Initial center frequencyguided VMD for fault diagnosis of rotating machines," Journal of Sound and Vibration, vol. 435, pp. 36-55, 2018.

[24] X. Zhang, Q. Miao, H. Zhang, and L. Wang, "A parameteradaptive VMD method based on grasshopper optimization algorithm to analyze vibration signals from rotating machinery," Mechanical Systems and Signal Processing, vol. 108, pp. 58-72, 2018.

[25] J. Zhu, C. Wang, Z. Hu, F. Kong, and X. Liu, "Adaptive variational mode decomposition based on artificial fish swarm algorithm for fault diagnosis of rolling bearings," Proceedings of the Institution of Mechanical Engineers, Part C: Journal of Mechanical Engineering Science, vol. 231, no. 4, pp. 635-654, 2017.

[26] X. L. Zhao, P. F. Wu, and X. X. Yin, "A quadratic penalty item optimal variational mode decomposition method based on single-objective salp swarm algorithm," Mechanical Systems and Signal Processing, vol. 138, Article ID 106567, 12 pages, 2020.

[27] T. Zhang, W. Chen, and M. Li, "AR based quadratic feature extraction in the VMD domain for the automated seizure detection of EEG using random forest classifier," Biomedical Signal Processing and Control, vol. 31, pp. 550-559, 2017.

[28] S. Mirjalili, S. M. Mirjalili, and A. Lewis, "Grey wolf optimizer," Advances in Engineering Software, vol. 69, pp. 46-61, 2014.
[29] A. Khan, S. Rehman, M. Abbas, and A. Ahmad, "On the mutual information of relaying protocols," Physical Communication, vol. 30, pp. 33-42, 2018.

[30] L. R. Quitadamo, R. Lucia, F. Gozzo et al., "Kurtosis-based detection of intracranial high-frequency oscillations for the identification of the seizure onset zone," International Journal of Neural Systems, vol. 28, no. 7, Article ID 1850001, 12 pages, 2018.

[31] S. Wade and R. Robert, "Rolling element bearing diagnostics using the Case Western Reserve University data: a benchmark study," Mechanical Systems and Signal Processing, vol. 64-65, pp. 100-131, 2015. 